\title{
Design of Terminal-independent Service Mobility in Wired and Wireless IP Network
}

\author{
Sunghyun Yoon, Tae Yeon Kim \\ Network Research Division, Electronics and Telecommunications Research Institute, 218 Gajeong-ro, Yuseong-gu, \\ Daejeon 34129, KOREA \\ *Corresponding Author: shy72@etri.re.kr
}

\begin{abstract}
Terminal-independent service mobility is capability to keep using a service while switching between terminals without service disconnection. That allows users to continue the active session between different terminals. Recently Internet keeps evolving toward ubiquitous environment. Users may use a lot of terminals for a particular IP (Internet Protocol) network service and choose the best terminal between user's available terminals depend on the situation. In this paper, we propose a TCS (terminal control server) concept for terminal-independent service mobility. The TCS enables users to choose the best terminal which fits ongoing service among available user terminals. For implementation, the TCS manages various information including user terminals and service subscription. This information mainly obtained from existing system such like AAA (Authentication Authorization Accounting) including authentication procedure and user profiles.
\end{abstract}

Keywords: Terminal-independent, Service mobility, IP network.

\section{Introduction}

The mobility is one of the main concerns in IP network. Internet standard organizations provide significant effort to implement the IP mobility in Internet such as MIPv4 (Mobile IP) ${ }^{(1,2)}$, MIPv6 (Mobile IPv6) ${ }^{(3)}$, PMIP (Proxy Mobile IP) $^{(4-6)}$ and NEMO (Network Mobility) ${ }^{(7)}$. Although many different mobility approaches are proposed, most approaches just provide solutions based on terminal dependent technologies. Using the Internet service, especially multimedia service, users may want to use more suitable terminal for better service quality ${ }^{(8-12)}$.
In general, devices using an IP (Internet protocol) service are terminals which can use an Internet service, for example, a mobile PC (Personal Computer), an IPTV (Internet Protocol television), and so on. These terminals provide users with IP services by using their own functions suitable for corresponding services. It tends to merge or combine functions of different terminals to produce a multifunctional terminal. For example, the multi-functional terminals include a mobile PC which enables a user to enjoy various IP services such as VoD (Video on Demand) and IPTV, and a mobile phone capable of Internet browsing.

However, even the multi-functional terminals that provide various functions have their own primary functions and additional secondary functions. For example, a terminal suitable for a voice-based service is preferably compact and light, like a mobile phone, so that a user can easily carry it, and a terminal suitable for a video service requires a large screen and high performance speakers in consideration of visual effects and sound effects.

The satisfaction of the user is proportional to how much a function of the terminal is specialized for the service, and thus the user tends to want a specialized terminal for a particular service, with a better performance than that of a secondary function of a multifunctional terminal. For example, if a user uses an IPTV service with a mobile phone while on the move, the user might want to keep using the IPTV service by changing a terminal from the mobile phone to a television with a bigger screen and a clear image quality when there is the television the user can use.

As mentioned above, when a user wants to keep using the same service through a different terminal, conventionally, the current used service is stopped and then 
the user is required to access to the same service using the new terminal. However, in such the method, a different service session needs to be generated for the newly started service, and thus the service continuity is not guaranteed.

In this paper, we design a TCS (terminal control server) which allows users to keep using a currently used IP service with a different terminal that the user owns without disconnection.

\section{Terminal Control Server}

The TCS is comprised of several sub-modules and each of them has their own functions respectively. Figure 1 shows the functional architecture of TCS. The TCS includes an AAA (authentication authorization accounting) interface unit, a terminal interface unit, a service/user property management unit, a terminal property management unit, a service/user status management unit, a terminal status management unit, a service proxy unit and a terminal-shifted service control unit.

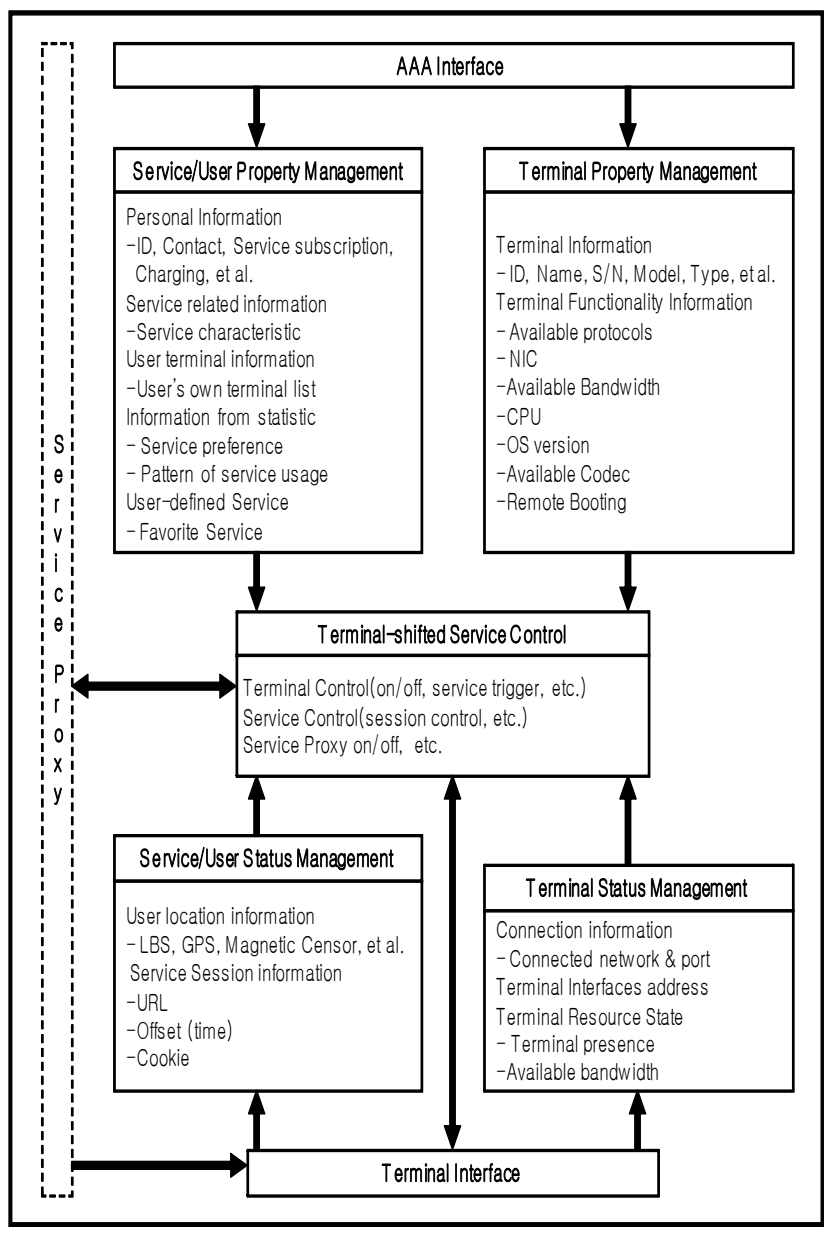

Fig. 1. Title. Functional architecture of TCS
The AAA interface unit receives unique information having fixed properties with respect to the terminal authorized by the AAA and the user of the terminal, and the two property (service/user, terminal) management units store and manage the unique information received from the AAA interface unit.

The unique information having fixed properties can be classified into terminal unique information and service and user unique information.

The terminal unique information includes general information of the terminal, such as, a terminal identification, a terminal name, a terminal serial number, a model of the terminal, and a shape of the terminal, and information of terminal's function, such as built-in protocol list, NIC maximum access speed, supportable bandwidth, CPU performance, an operating system version, compatible codec, and remote control capability. These terminal related properties are managed by the terminal property management unit, which enables a terminal-shifted service control unit to recognize what service can be provided to a corresponding terminal.

The service and user unique information includes user personal information (e.g., identification, contact numbers, subscribed services, bills and the like), general information relating the services (i.e., service feature information) and information of user's terminal (a list of owned terminals). Such the service and user related properties are stored and managed by a service/user property management unit, which infers connection between pieces of information, thereby obtaining a user's taste in services, a pattern of use, and service preference of the user.

Thus, the terminal-shifted service control unit can learn through the service/user property management unit what service the user has subscribed to, kinds and features of the services that are provided to the user, what types of valid terminals the user owns, and the service preference of the user.

The terminal interface unit receives status information having variable properties with respect to an authorized terminal and the currently used service from the authorized terminal, and two status management units store and manage the pieces of status information received from the terminal interface unit.

The service/user status management unit manages the status information, such as user's location information and service session information, which varies according to the status of use of the service. Also, the service/user status management unit performs reset or synchronization on the 
currently used service, thereby managing the status of the current service. By the service/user status management unit, the terminal-shifted service control unit can recognizes what service can be provided to the terminal based on the current location of the user, the status of the currently used service, and if the shift in the terminals is available for the currently used service.

The terminal status management unit manages the status information of each terminal that the user has registered. The status information of various terminals registered by the user includes pieces of variable information such as an access network, access port information, a terminal address, terminal source availability which varies according to use of the service. The variable information is periodically obtained from a corresponding terminal and updated.

The terminal-shifted service control unit primarily controls the terminal-shifted service. When a terminal that is using a service requests the TCS to shift the service to different terminal, the terminal-shifted service control unit refers to the (unique and status) information of the user and the service and provides the requested terminal with information of terminals available for the service based on the information, and generates service session information and sends it to a selected terminal for shifted service.

The service proxy unit requests the CP (contents provider) to send the service that the requested terminal is using, receives service data in response, and modifies the service data in accordance with the specification of the terminal to be provided with the service and then sends the modified service data to the terminal. The service proxy unit acts as a buffer between the terminal that requests the shift of the service and the terminal to which the service is shifted.

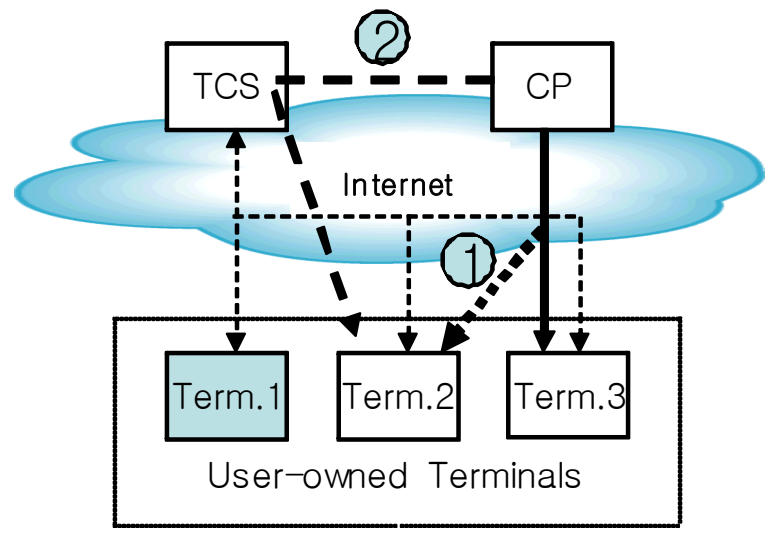

Fig. 2. Terminal-shifted Service

\section{Terminal-shifted Service}

For the terminal-independent service mobility, the TCS manages pieces of information which are classified into information having fixed properties and information having variable properties according to variability in use of the IP services.

The information having fixed properties may be general information of the user and information about the subscribed service. Generally, such the information is already possessed by a service provider, and is utilized for the AAA or the like. Meanwhile, the information having variable properties may be information regarding the terminal or service session information, which changes according to the use of the service. Unique information that has fixed properties can be obtained through the AAA which is a subscriber terminal authentication system, and the information having variable properties can be obtained from each terminal. Figure 2 shows concept of how to shift a service from one terminal to a different terminal while a user is using a service.

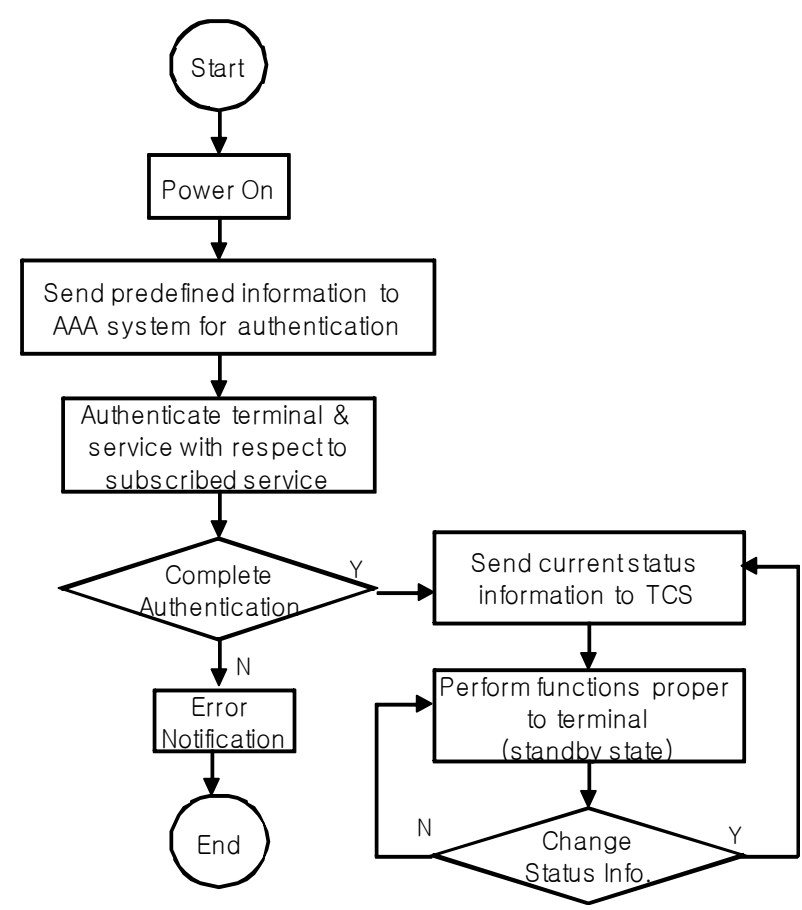

Fig. 3. Terminal operation for TCS

Referring to Figure 2, the terminal-shifted service may be classified into two types according to whether the TCS modifies the service data. One is to use mainly the terminal-shifted service control unit, and another is to use the service proxy unit of TCS.

In the first type, the TCS obtains unique and status 
information of each user-owned terminal, which are registered by a user through an IP network, and retains the information. When the user requests to shift the IP service from the terminal 3 to terminal 2, the TCS controls terminal 2 to issue a service request to the $\mathrm{CP}$ by utilizing the service status information which has been obtained from terminal3. Then the CP provides terminal 2 with a service to which a corresponding service session is applied, and stops the service to terminal 3.

In the second type, a terminal-shifted service can be provided using a service proxy unit. The TCS requests the $\mathrm{CP}$ to send service data when there is a terminal shift request from a user after the user registers a service and information of the terminal that the user is using through an IP network. Then the TCS receives the requested service data, processes the data in accordance with the specification of a terminal which is requested to be provided with the service, i.e. term.2, and then sends the modified data to the terminal.

Figure 3 shows terminal operation for TCS. When a terminal is turned on, the terminal sends authentication information to the AAA system for the authentication process of service provider, and the AAA system processes the authentication for the terminal and IP service based on the authentication information sent from the terminal, and notifies the terminal of the completion of authentication. Once the authentication is verified, the user terminal is authorized to use the IP service. Such the authentication procedures are defined by unique authentication processes of the each service provider. After the authentication process, the terminal which is informed of authentication completion from the AAA sends information about the current status of the terminal, including an address, network information and service usage information, to the TCS by periods. The TCS stores and manages pieces of status information received from individual terminals, thereby capable of learning the current status of the corresponding terminal.

Figure 4 shows service shift procedure using TCS. Referring to Figure 4, an ongoing service is shifted from previous terminal to new terminal.

First, the authentication process predetermined by a service provider is performed, and the AAA sends TCS obtainable unique information such as property of the terminal, property of the terminal user and the information of the terminal the user is holding when the authentication is complete according to the predefined procedures.

The authenticated terminal can access the service (including network service, value added IP service), and the terminal sends the status information regarding the current service and the terminal to the TCS. These procedures can be referred to as preparation for use of the service of each terminal. When the preparation for use of the service with respect to each terminal is completed, the service/user property management unit and terminal property management unit of the TCS stores and manages the unique information of the service and the user and the unique information of the terminal respectively. Likewise, the service/user status information management unit and terminal status management unit stores the status information of the service and the user and the terminal status information respectively. Then the terminal issues a service request to the $\mathrm{CP}$ and the $\mathrm{CP}$ provides the requested service to the terminal.

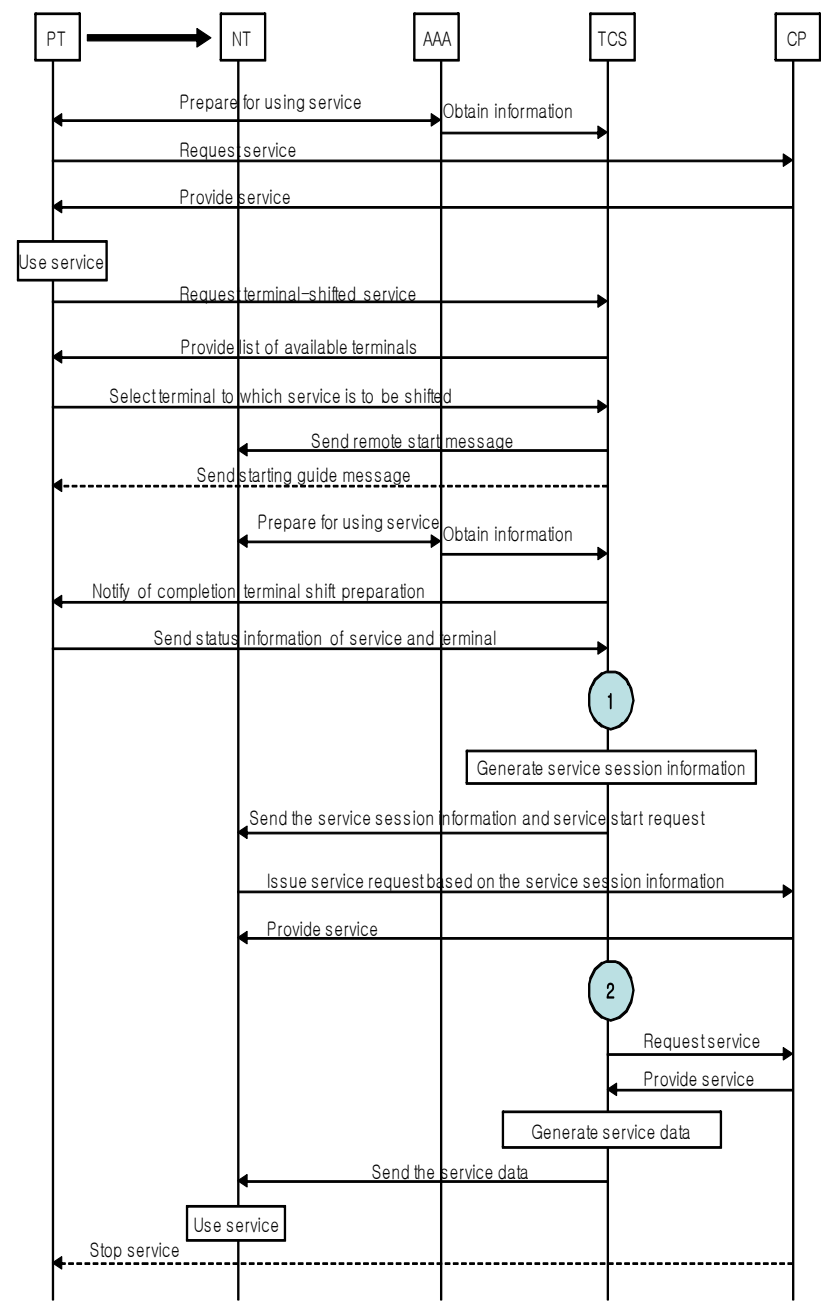

Fig. 4. Service Shift Procedure

Thereafter, if the user needs to change the terminal for use of the service with a new terminal while using the service with the previous terminal, the user can request the 
TCS for the terminal-shifted service. A particular button or switch may preferably be used for this procedure to initiate a specific function to interrupt the operation of the terminal which is using the service.

Receiving the request of the terminal-shifted service, the TCS searches the each property management unit, and makes a list of terminals that have been registered by the user and are available for the current IP service (the list includes only available terminals, except the offline terminal like the terminal 1 in Figure 2), and sends the list to the previous terminal on the user side. In response, the user chooses a terminal from the list and sends the user's selection to the TCS.

Then, the TCS sends the selected terminal a remote start message in accordance with the characteristics of the selected terminal, and remotely starts the selected terminal (i.e. new terminal). Alternatively, the TCS sends the user a starting guide message so that the user can start the desired terminal manually. However, if the new terminal has been already turned on, the TCS is aware of this situation, and thus the procedure will be discarded. And then, the preparation of use of the service is performed on the new terminal to which the service is to be shifted. When the new terminal is ready for using the service, the TCS sends a preparation completion message to the previous terminal.

The previous terminal that receives the message sends the status information of the current using service and the terminal status information to the TCS. The service status information to be sent may be a kind of the current service and the method of accessing to the service, cookie information including the service log-on information and temporary information generated in the course of using the service, and service usage information including the time when the service is used or the progress of the service. In addition, the terminal status information may be an IP address obtained by the terminal, information of the currently used access network, resource usage information including the bandwidth and CPU occupancy rate, etc.

Hereafter, the rest of the procedure can be separated into two types of process according to using the service proxy unit of TCS.

In the first type process, the TCS receives the status information as mentioned above, generates service session information regarding the current service for the new terminal, and sends the generated service session information along with a service starting request to the new terminal. In response, the new terminal issues a service request to the $\mathrm{CP}$ based on the service session information received from the TCS, and the $\mathrm{CP}$ provides the corresponding service to the new terminal in response to the service request from the new terminal.

Thus, according to the procedures described above, for example, when a user who is doing Internet shopping using a desktop personal computer (previous terminal) at home wants to go out, the user can continue to browse the Internet outside the home using a laptop computer (new terminal) by changing the terminal to the laptop computer.

In the second type process, the service proxy unit of the TCS requests the $\mathrm{CP}$ to send the service that the previous terminal is currently using. When the CP sends the service data to the TCS in response, the service proxy unit processes the corresponding service data in accordance with the status of the new terminal. The service data may be processed to have its bandwidth or codec adjusted, or to have offset information applied thereto. The processed data service is sent to the new terminal.

Thereafter, the service provided to the previous terminal can be stopped according to the type of the service policies of the $\mathrm{CP}$, and the stopping time point may vary according to the policies of the CP.

After all process so far, the user can use the service through the new terminal. That is, for example, a user who is using a video streaming service through a PDA outside home can change the terminal to an IPTV at home to continue to use the video streaming service without disconnection.

\section{Conclusions}

Due to the continuous and explosive growth of wireless Internet, mobility has become a fundamental attribute of current communication environment. However, mobility management technology still depends on terminal mobility.

On the one hand, it is predicted that the IoT era will come in the not too distant future. At that time, the current communication environment will have many changes. In the end, it will evolve into an environment that allows individual users to receive desired services easily and at anytime and anywhere through a variety of terminals, rather than being able to receive various services through a single terminal.

In this paper, we proposed TCS concept and designed a functional architecture of the TCS to provide terminal-independent service mobility, which enables users to use the terminal-shifted service between user's terminals 
in Internet. Accordingly, user can conveniently change the terminal to use seamless service without disconnection. This availability encourage the user to use a terminal the most suitable for ongoing service from among terminals that the user has owned and registered, and thus the user can obtain more satisfaction regarding the use of the service.

Also, most fixed property information (i.e. service, user, terminal related information) from among information about items to be managed by the TCS can be obtained through the existing system such like AAA. The terminal-independent service mobility can be implemented by only adding a TCS system to a network and adjusting software of the existing terminal operation and an AAA authentication system.

\section{Acknowledgment}

This research was partially supported by the IT R\&D program of MSIT (Ministry of Science and ICT) [2018-0-00226, Development of Trusted Reality core technology for the era of post smartphone]

\section{References}

(1) C. Perkins : "IP Mobility Support for IPv4, Revised", IETF RFC 5944, November 2010

(2) C. Perkins, P. Calhoun, J. Bharatia : "Mobile IPv4 Challenge/Response Extensions (Revised)", IETF RFC 4721, January 2007

(3) C. Perkins, D. Johnson, J. Arkko : "Mobility Support in IPv6", IETF RFC 6275, July 2011

(4) S. Gundavelli, K. Leung, V. Devarapalli, K. Chowdhury, B. Patil : "Proxy Mobile IPv6", IETF RFC 5213, August 2008

(5) S. Gundavelli : "Reserved IPv6 Interface Identifier for Proxy Mobile IPv6", IETF RFC 6543, May 2012

(6) CJ. Bernardos : "Proxy Mobile IPv6 Extensions to Support Flow Mobility", IETF RFC 7864, May 2016

(7) V. Devarapalli et al. : "Network Mobility(NEMO) Basic Support Protocol", RFC3963, 2005.

(8) W. Munkongpitakkun et al. : "Enhanced Web Session Mobility based on SIP", Mobility 2007, pp.346-350.

(9) H. Mukhtar et al. : "Session Mobility of Multimedia Applications in Home Networks Using UPnP", INMIC, 2007.

(10) Y. Lee et al. : "UMOST : Ubiquitous Multimedia Framework for Context-aware Session Mobility",
ICMUE, April 2008.

(11) H. Li, C. Peng, B. Li, Y. Chen, W. Zhang, J. Wu, and H. Huang : "User ID routing architecture", IEEE Veh. Technol. Mag., Vol.5, No. 1, pp. 62-69, Mar. 2010.

(12) Y. Cui, K. Nahrstedt, and D. Xu : "Seamless user-level handoff in ubiquitous multimedia service delivery", ACM Multimedia Tools and Appl., Vol. 22, No. 2, pp. 137-170, Feb. 2004. 